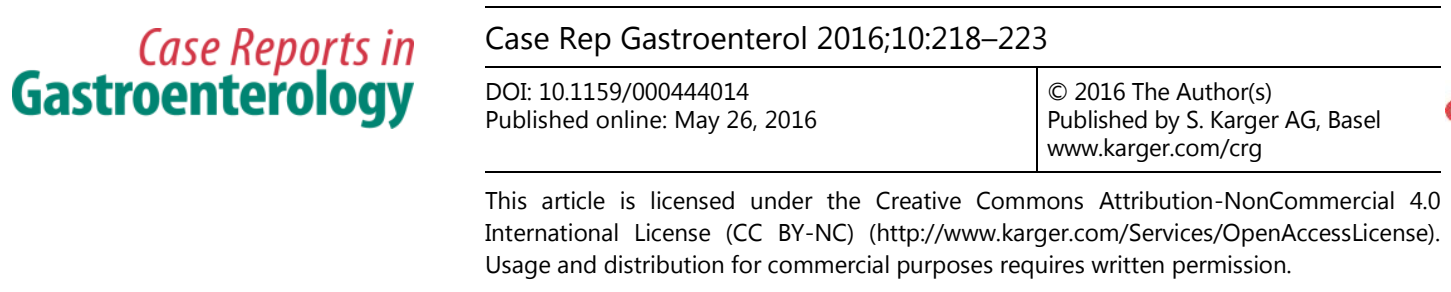

\title{
Unintentional Long-Term Esophageal Stenting due to a Complete Response in a Patient with Stage UICC IV Adenocarcinoma of the Gastroesophageal Junction
}

\author{
Anna Paeschke ${ }^{a} \quad$ Christian Bojarski $^{a} \quad$ Susanne Küpferling ${ }^{b}$ \\ Thomas Hucklenbroich $^{c}$ Britta Siegmund ${ }^{a}$ Severin Daum ${ }^{a}$ \\ ${ }^{a}$ Department of Gastroenterology, Infectious Diseases and Rheumatology, Campus \\ Benjamin Franklin, Charité - Universitätsmedizin Berlin, Berlin, Germany; ${ }^{b}$ Department for \\ Gastroenterology, Vivantes Wenckebach-Klinikum, Berlin, Germany; \\ ${ }^{\mathrm{c}}$ Gastroenterologische Praxis, Berlin, Germany
}

\section{Keywords}

Endoscopic stent implantation - Adenocarcinoma of the esophageal junction and stomach . Long-time survival

\begin{abstract}
Endoscopic stent implantation is a common short-treatment option in palliative settings in patients with esophageal cancer. Advanced disease is associated with low survival rates; therefore, data on the long-term outcome are limited. So far, cases of long-term remission or even cure of metastasized adenocarcinoma of the gastroesophageal junction or stomach (AGS) have only been reported from Asia. A 51-year-old male patient primarily diagnosed with metastasized adenocarcinoma of the gastroesophageal junction (GEJ) [type I, cT3cN+cM1 (hep), CEA positive, UICC stage IV] received palliative esophageal stenting with a self-expandable metal stent. As disease progressed after four cycles with epirubicin, oxaliplatin, and capecitabin, treatment was changed to 5-FU and Irinotecan. The patient did not return after 5 cycles of FOLFIRI, but presented 4 years later with mild dysphagia. Endoscopy surprisingly revealed no relevant stenosis or stent migration. Repeated histological analyses
\end{abstract}


of a residual mass at the GEJ did not detect malignancy. Since the initially diagnosed hepatic metastases were no longer detectable by computed tomography, cure from esophageal cancer was assumed. Dysphagia was ascribed to esophageal motility disorder by a narrowed esophageal lumen after long-term stenting. Thus, endoscopic stent implantation is an important method in palliative treatment of dysphagia related to AGS. New systemic treatment strategies like trastuzumab in Her2neu positive cases or new VEGF-inhibitors like ramucirumab will lead to more long-time survivors with AGS. In conclusion, future endoscopic treatment strategies in AGS represent a challenge for the development of new stent techniques in either extraction or programmed complete dissolution.

(C) 2016 The Author(s)

Published by S. Karger AG, Basel

\section{Introduction}

Esophageal cancer represents a serious malignancy and is associated with high mortality. Among the different histological subtypes, squamous cell carcinoma (SCC) and adenocarcinoma represent over $95 \%$ of all esophageal carcinomas. Whereas prevalence of SCC has decreased over the past decades, rising incidence of adenocarcinoma has been observed [1]. This has been partially attributed to the higher occurrence of risk factors like obesity and gastroesophageal reflux disease in Western countries. Whereas obesity is thought to promote tumor development and progression by pro-inflammatory cytokines, reflux supports the replacement of the regular stratified squamous epithelium lining of the esophagus by simple columnar epithelium with goblet cells. The so-called Barrett metaplasia is a precancerous condition [2].

Adenocarcinomas of the esophagus are associated with poor prognosis. The 5-yearsurvival is below $20 \%$ [3]. Only localized disease with early TNM-stages like Tis, T1 or T2 carcinoma are open for surgical treatment strategies without preoperative chemo- or radiotherapy [4]. However, over $50 \%$ of all patients have evidence of distant metastases at the time of initial diagnosis [2]. In these cases, therapeutic options are limited to palliative chemotherapy, radiochemotherapy and best supportive care. The leading cause for hospitalization of patients with advanced disease is dysphagia, due to stenosing tumor formation. In these cases, supportive care includes endoscopic stent implantation to restore luminal patency and thereby enabling oral food intake and improving quality of life. Self-expandable metal stents (SEMS) represent the state of the art in endoscopic stent implantation. Especially patients with reduced life expectancy benefit from this treatment option and often experience a rapid symptom relief within 24-48 h [5]. Typical long-term complications (6 weeks after implantation) are pain, bleeding, development of esophagotracheal fistula or recurrent dysphagia due to food bolus impaction or tumor in- and overgrowth and necessitate reinterventions [6].

We report a case of complete remission of advanced metastasized adenocarcinoma of the gastroesophageal junction (AGS) and unintentional long-term esophageal stenting.

\section{Case Presentation}

A 51-year-old man initially presented to our institution in 2011. He had experienced progressive dysphagia and weight loss in the 3 months prior to hospitalization. Food or even liquid intake was no longer possible. Esophagogastroduodenoscopy disclosed a stenosing 
tumor at a $30-\mathrm{cm}$ distance to the dental arch. The remaining lumen was restricted to about $3 \mathrm{~mm}$, impeding further investigation by endosonography. Histological examination revealed a moderately differentiated adenocarcinoma. The tumor marker carcinoembryonic antigen (CEA) was slightly increased $(14.4 \mu \mathrm{g} / \mathrm{l})$. Further staging by abdominal and thoracic computed tomography (CT) showed various prominent mediastinal lymph nodes and a single hepatic lesion in segment IVb, which was radiologically rather interpreted as a hemangioma than a metastasis, indicating at least a UICC stage III carcinoma (fig. 1a). The patient received tube feeding as supportive therapy via a surgically placed jejunal catheter. As recommended by the Interdisciplinary Tumor Board of our institution, neoadjuvant chemotherapy with epirubicin, oxaliplatin and capecitabin (EOX, epirubicin $50 \mathrm{mg} / \mathrm{m}^{2}$, oxaliplatin $130 \mathrm{mg} / \mathrm{m}^{2}$, capecitabin $1,250 \mathrm{mg} / \mathrm{m}^{2}$ ) was initiated. Restaging after four cycles revealed progressive disease to UICC stage IV, as multiple new metastatic lesions with a maximal diameter of $6 \mathrm{~mm}$ in the liver and an increase of CEA $(74.5 \mu \mathrm{g} / \mathrm{l})$ were detected (fig. 1b). Therefore, the chemotherapy protocol was changed to administration of five cycles of FOLFIRI (5-FU 2,000 $\mathrm{mg} / \mathrm{m}^{2}$, folinic acid $500 \mathrm{mg} / \mathrm{m}^{2}$, irinotecan $80 \mathrm{mg} / \mathrm{m}^{2}$ ), and supportive care was complemented by endoscopic stent implantation (SEMS Leufen ${ }^{\circledR}$, partially silicone covered, length $14 \mathrm{~cm}$, diameter $2 \mathrm{~cm}$ ).

Four years later, the patient was admitted with dysphagia and deterioration of the general condition to another hospital. In the meantime, no follow-up examinations by endoscopy or CT had been undertaken. Now, current restaging by CT did not reproduce the prediagnosed hepatic metastases (fig. 1c). Endoscopy of the upper gastrointestinal tract surprisingly disclosed no relevant stenosis or stent migration (fig. 2). Functional examination by gastrographin X-ray confirmed an undisturbed swallowing function and esophageal passage. Histological processing of the biopsy samples of the residual mass at the GEJ displayed no malignancy but chronic inflammation. The resulting reactive scar tissue formation was suspected to cause the described dysphagia. In summary, this implicates a complete response of an initially UICC stage IV adenocarcinoma of the esophagus to chemotherapy with five cycles of FOLFIRI. The esophageal stent, which was implanted in the context of supportive care to allow oral food intake in the suspected residual life span, remained for at least 4 years in situ without major complications such as bleeding, development of fistulas or stent migration. However, present dysphagia was explained by esophageal motility disorder due to narrowing of the esophageal lumen after long-term stenting rather than relevant stenosis because of scar tissue formation. Therefore, the stent was left in situ, as stent removal of the completely ingrown stent was supposed to be associated with high risk of bleeding and perforation.

\section{Discussion}

We report for the first time a case of a 51-year-old Caucasian man with advanced adenocarcinoma of the GEJ who showed a complete response to a second-line therapy. In expectancy of a short remaining lifetime, palliative chemotherapy was complemented by endoscopic stent implantation to overcome dysphagia and malnutrition due to stenosing tumor formation. Unexpectedly, palliative chemotherapy with FOLFIRI led to a complete response.

Esophageal cancer is associated with a 5-year survival rate below $20 \%$, which is attributable to the frequently observed advanced stage of disease at the time of diagnosis. Recommended treatment strategies depend on the current TNM stage. In localized disease with T stages of Tis, $\mathrm{T} 1$ or $\mathrm{T} 2$, local ablation or surgery are the preferred treatment options. 
In terms of lymph node metastases, this may be combined with neoadjuvant chemotherapy. Whenever distant metastases occur, palliative chemotherapy or radiochemotherapy shall be administered and may be complemented with best supportive care [7].

Second line chemotherapy with irinotecan, as was done in our case, has been proven to be effective, and recent phase III data even demonstrated superior efficacy of FOLFIRI over ECX as first-line therapy in advanced gastric and EGJ cancer $[8,9]$. In spite of this activity of irinotecan, cases with complete response to chemotherapy of advanced AGS have only been reported from Asia [10-12].

In our case, due to the unexpected complete response, the stent 'accidentally' remained in situ for at least 4 years without major complications. Endoscopic stent implantation is an important tool in palliation of gastrointestinal cancer to overcome tumor-associated stenoses. Due to the continuous development over the time, a broad spectrum of different stent types with specific advantages and disadvantages has emerged. SEMS represent the state of the art in endoscopic stent implantation.

Complications of stent implantation include acute complications like organ perforation, tracheal compression or malposition as well as delayed complications like stent dislocation or bleeding. Severe complications often occur at a later stage (over a week after stent implantation) and comprise recurrence of dysphagia due to tumor overgrowth or food impaction, stent migration, development of tracheoesophageal fistula or gastroesophageal reflux. Therefore, this method should be reserved for palliative settings and patients with a short remaining life expectancy, which limits data on long-term experiences. A study published by Schoppmann et al. [13] monitored 70 patients who received stent implantation for different benign and malignant esophageal disorders. Stents remained in situ for a mean time of 13.5 \pm 11.8 months (median 294 days). The overall complication rate was about $30 \%$. They observed low rates for stent-related complications like migration (14\%), perforation $(1.4 \%)$ and bleeding $(0 \%)$. These complications were independent of stent characteristics like length, diameter, material or coating, providing evidence that long-term stenting is safe and effective. However, there are currently no recommendations for timely controlled stent removal or regular follow-up intervals available in a palliative setting.

\section{Conclusion}

Endoscopic stent implantation is an important tool to overcome dysphagia related to advanced esophageal or gastric cancer in patients with a short remaining life expectancy. Here, we describe a case with unintentional esophageal stenting for about 4 years, due to an unexpected completed response of an advanced adenocarcinoma of the GEJ to chemotherapy. In the next years, new systemic treatment strategies like trastuzumab in Her2neupositive cases or new VEGF-inhibitors like ramucirumab will hopefully increase the number of long-term survivors of AGS $[14,15]$.

In conclusion, as demonstrated by this case, future hopefully improved treatment strategies in AGS represent a challenge for the development of new stent techniques in either extraction or by placement of biodegradable stents.

\section{Statement of Ethics}

The patient has given his informed consent to publication. 


\section{Case Reports in \\ Gastroenterology}

\begin{tabular}{l|l}
\hline $10.1159 / 000444014$ & $\begin{array}{l}\text { (c) } 2016 \text { The Author(s). Published by S. Karger AG, Basel } \\
\text { www.karger.com/crg }\end{array}$ \\
\hline
\end{tabular}

Paeschke et al: Unintentional Long-Term Esophageal Stenting due to a Complete Response in a Patient with Stage UICC IV Adenocarcinoma of the Gastroesophagea Respons

\section{Disclosure Statement}

There is no conflict of interest to declare.

\section{References}

1 Absi A, Adelstein DJ, Rice T: Esophageal cancer. Cleveland Clinic, 2013.

http://www.clevelandclinicmeded.com/medicalpubs/diseasemanagement/hematology-

oncology/esophageal-cancer/.

-2 Enzinger PC, Mayer RJ: Esophageal cancer. N Engl J Med 2003;349:2241-2252.

-3 Dubecz A, Gall I, Solymosi N, et al: Temporal trends in long-term survival and cure rates in esophageal cancer: a SEER database analysis. J Thorac Oncol 2012;7:443-447.

4 Sancheti M, Fernandez F: Management of T2 esophageal cancer. Surg Clin North Am 2012;92:11691178.

-5 Homs MY, Steyerberg EW, Eijkenboom WM, et al: Single-dose brachytherapy versus metal stent placement for the palliation of dysphagia from oesophageal cancer: multicentre randomised trial. Lancet 2004;364:1497-1504.

-6 Larssen L, Medhus AW, Korner H, et al: Long-term outcome of palliative treatment with self-expanding metal stents for malignant obstructions of the GI tract. Scand J Gastroenterol 2012;47:1505-1514.

7 Napier KJ, Scheerer M, Misra S: Esophageal cancer: a review of epidemiology, pathogenesis, staging workup and treatment modalities. World J Gastrointest Oncol 2014;6:112-120.

-8 Guimbaud R, Louvet C, Ries P, et al: Prospective, randomized, multicenter, phase III study of fluorouracil, leucovorin, and irinotecan versus epirubicin, cisplatin, and capecitabine in advanced gastric adenocarcinoma: a French intergroup (Federation Francophone de Cancerologie Digestive, Federation Nationale des Centres de Lutte Contre le Cancer, and Groupe Cooperateur Multidisciplinaire en Oncologie) study. J Clin Oncol 2014;32:3520-3526.

-9 Thuss-Patience PC, Kretzschmar A, Bichev D, et al: Survival advantage for irinotecan versus best supportive care as second-line chemotherapy in gastric cancer-a randomised phase III study of the Arbeitsgemeinschaft Internistische Onkologie (AIO). Eur J Cancer 2011;47:2306-2314.

10 Nakashima S, Hatanaka N, Yoshikawa Y, et al: Long-term survival of a case of advanced cancer of the esophagogastric junction with complete response to low-dose 5-fluorouracil and cisplatin chemotherapy (in Japanese). Gan To Kagaku Ryoho 2012;39:1559-1561.

11 Nishino T, Yamamoto Y, Ikeda M, et al: Pathological complete response in a case of advanced esophageal cancer invading aorta treated by preoperative chemotherapy with docetaxel and cisplatin plus 5-FU (in Japanese). Gan To Kagaku Ryoho 2013;40:643-646.

12 Sasaki K, Natsugoe S, Aridome K, et al: The successfully curative treatment of advanced gastric adenocarcinoma with multiple liver metastases and paraaortic lymph node metastases by salvage operation following the biweekly paclitaxel and S-1 combination chemotherapy: a case report. Hepatogastroenterology 2007;54:2421-2424.

13 Schoppmann SF, Langer FB, Prager G, et al: Outcome and complications of long-term self-expanding esophageal stenting. Dis Esophagus 2013;26:154-158.

14 Bang YJ, Van Cutsem E, Feyereislova A, et al: Trastuzumab in combination with chemotherapy versus chemotherapy alone for treatment of HER2-positive advanced gastric or gastro-oesophageal junction cancer (ToGA): a phase 3, open-label, randomised controlled trial. Lancet 2010;376:687-697.

15 Fuchs CS, Tomasek J, Yong CJ, et al: Ramucirumab monotherapy for previously treated advanced gastric or gastro-oesophageal junction adenocarcinoma (REGARD): an international, randomised, multicentre, placebo-controlled, phase 3 trial. Lancet 2014;383:31-39. 


\section{Case Reports in Gastroenterology}

\begin{tabular}{l|l}
\hline Case Rep Gastroenterol 2016;10:218-223 \\
\hline $10.1159 / 000444014$ & $\begin{array}{l}\text { ○ 2016 The Author(s). Published by S. Karger AG, Basel } \\
\text { www.karger.com/crg }\end{array}$ \\
\hline
\end{tabular}
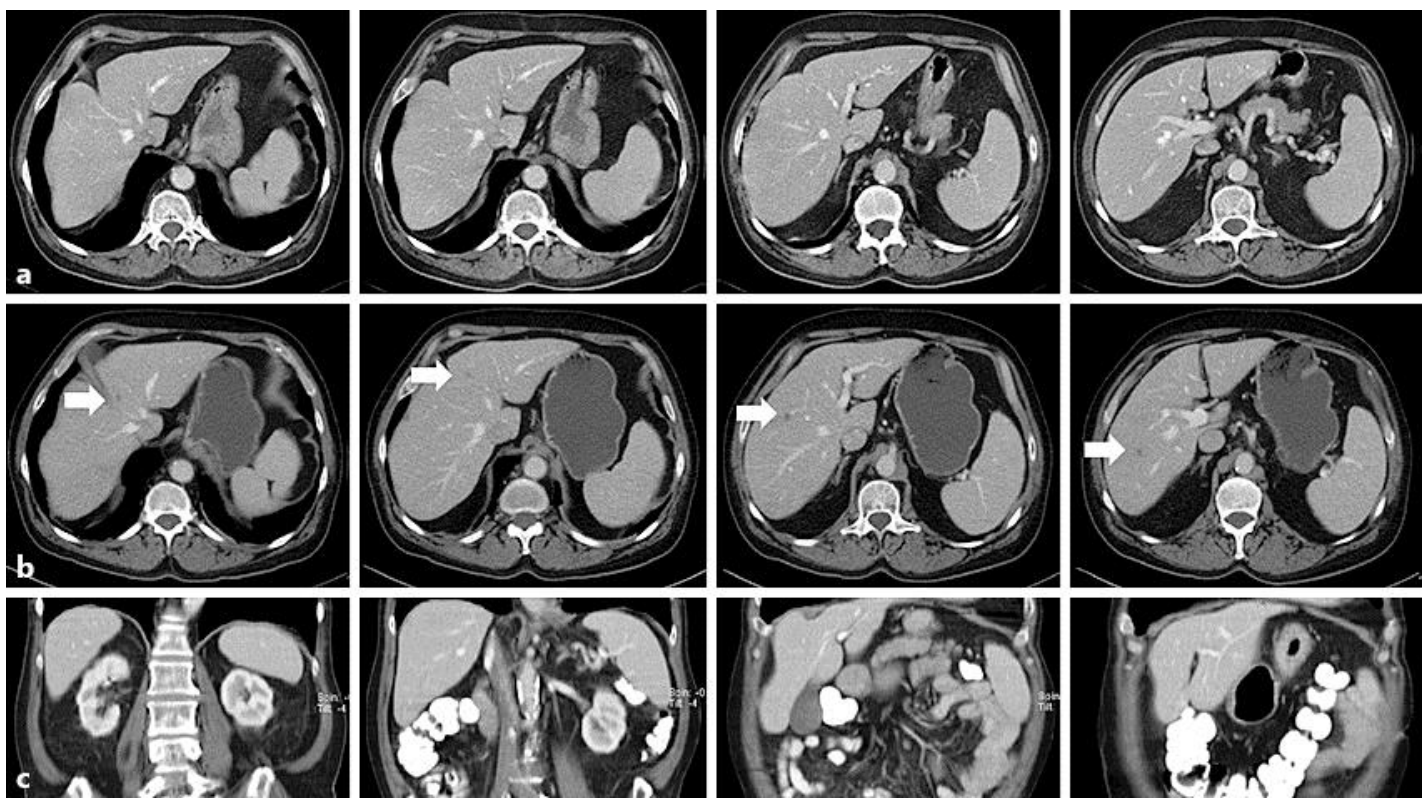

Fig. 1. a Initial abdominal CT scan prior to initiation of chemotherapy did not detect hepatic metastases. b After four cycles of EOX (epirubicin, oxaliplatin, capecitabin), CT staging indicated disease progression, as multiple metastatic lesions were detected (exemplarily indicated by white arrows). Therefore, the chemotherapy regimen was changed, and the patient received five cycles of FOLFIRI. c Four years later, restaging by abdominal CT scan did not show the former diagnosed metastatic lesions.

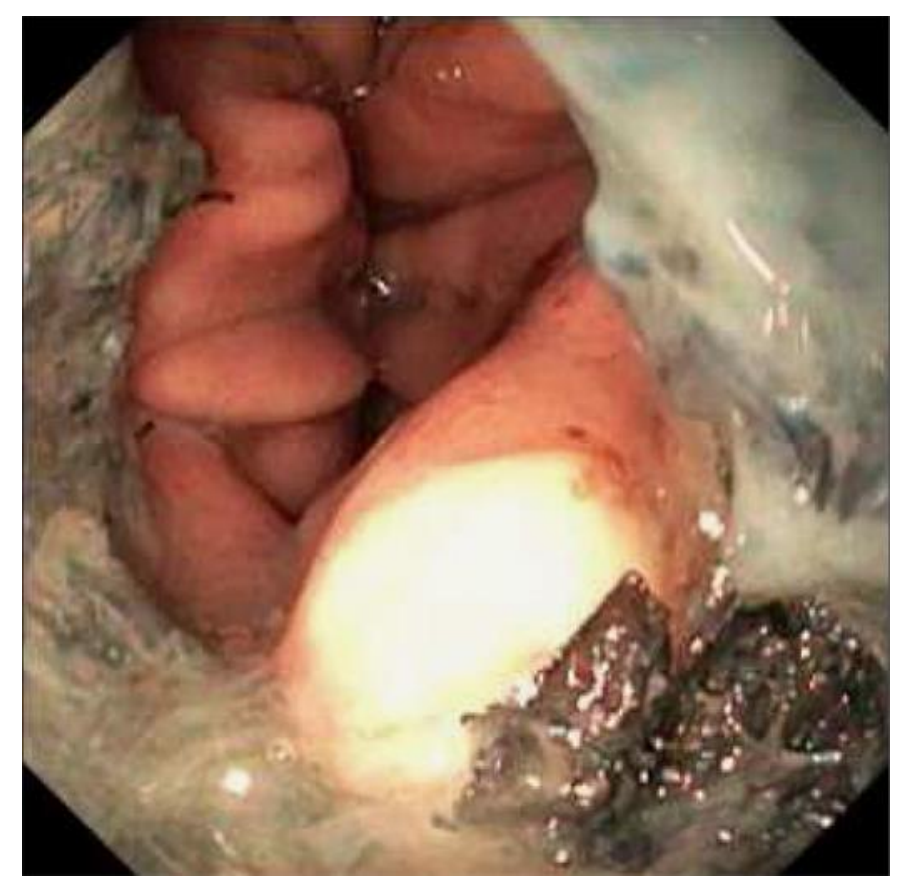

Fig. 2. Endoscopy of the upper gastrointestinal tract depicts the distal end of the non-dislocated stent after 4 years without further therapy. 